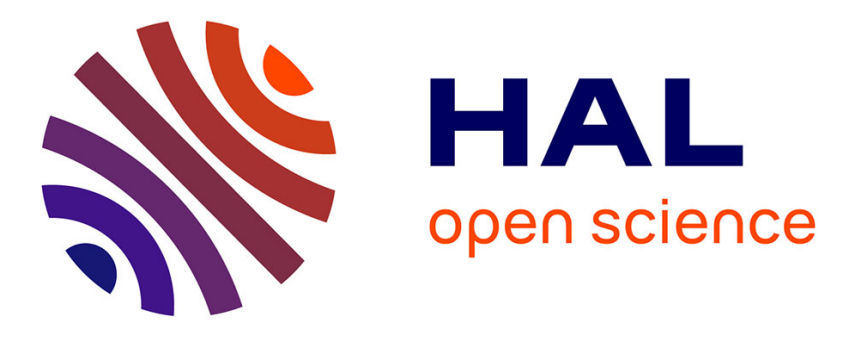

\title{
Unveiling the thermodynamic driving forces for high entropy alloys formation through big data ab initio analysis
}

Georgios B. Bokas, Wei Chen, Antoine Hilhorst, Pascal J. Jacques, Stéphane Gorsse, Geoffroy Hautier

\section{To cite this version:}

Georgios B. Bokas, Wei Chen, Antoine Hilhorst, Pascal J. Jacques, Stéphane Gorsse, et al.. Unveiling the thermodynamic driving forces for high entropy alloys formation through big data ab initio analysis. Scripta Materialia, 2021, 202, 114000 (5 p.). 10.1016/j.scriptamat.2021.114000 . hal-03263540

\author{
HAL Id: hal-03263540 \\ https://hal.science/hal-03263540
}

Submitted on 17 Jun 2021

HAL is a multi-disciplinary open access archive for the deposit and dissemination of scientific research documents, whether they are published or not. The documents may come from teaching and research institutions in France or abroad, or from public or private research centers.
L'archive ouverte pluridisciplinaire HAL, est destinée au dépôt et à la diffusion de documents scientifiques de niveau recherche, publiés ou non, émanant des établissements d'enseignement et de recherche français ou étrangers, des laboratoires publics ou privés. 


\title{
Unveiling the thermodynamic driving forces for high entropy alloys formation through big data $\mathrm{ab}$ initio analysis
}

\author{
G.B. Bokas ${ }^{\mathrm{a}}$, W. Chen ${ }^{\mathrm{a}}$, A. Hilhorst ${ }^{\mathrm{b}}$, P.J. Jacques ${ }^{\mathrm{b}}$, S. Gorsse ${ }^{\mathrm{c}}$, G. Hautier, ${ }^{\mathrm{a}, \mathrm{d}, *}$ \\ ${ }^{a}$ UCLouvain, Institute of Condensed Matter and Nanoscience (IMCN), Chemin étoiles 8, bte L7.03.01, Louvain-la-Neuve 1348, Belgium \\ ${ }^{b}$ UCLouvain, Institute of Mechanics, Materials and Civil Engineering, IMAP, Place Sainte Barbe 2, Louvain-la-Neuve 1348, Belgium \\ ${ }^{c}$ CNRS, University of Bordeaux, Bordeaux INP, ICMCB, UMR 5026, Pessac 33600, France \\ ${ }^{d}$ Dartmouth College, Thayer School of Engineering, Hanover 03755, NH, USA
}

\begin{abstract}
The fundamental thermodynamic driving forces beyond the existence of high entropy alloys (HEAs) are still not firmly understood. Here, using thermodynamic modeling combining ab initio computations with a regular solution model, we build a database of more than 100000 BCC and FCC equimolar alloys formed using 27 common elements. We statistically study how enthalpic and entropic contributions evolve with the number of elements in a random solid solution. The commonly admitted rationalization of a stabilization of HEAs due to a growing importance of the entropy with the number of elements is somewhat contradicted. Entropic and enthalpic contributions favor mixing in average, but both driving forces weaken as the number of elements in the alloy increases. By adding binary intermetallics to our analysis, we conclude that the specific chemical compositions prone to form single phase HEAs need to combine an enthalpically favorable mixing of their elements on a given lattice with the absence of strongly competing intermetallics.
\end{abstract}

*Author to whom correspondence should be addressed

Email address: geoffroy.hautier@uclouvain.be (G. Hautier) 
High-entropy alloys (HEAs) [1, 2] and the holistic view of complex concentrated alloys [3] have attracted great attention in recent years because they offer new degrees of freedom to design alloys with attractive combinations of properties [4, 5, 6, 7]. Contrary to traditional alloys which rely on one dominant base metal and small additions of other elements, HEAs are concentrated solutions of 5 or more principle elements forming single-phase solid solutions [8]. This represents a paradigm shift from the edges of the hyperdimensional compositional space to the vast and unexplored central regions [9]. It could look surprising that thermodynamics allows such singlephase multicomponent alloys to form instead of multiple phases. Indeed, numerous attempts of forming alloys at equiatomic compositions have led to multiphase microstructures and it is likely that only specific combinations of elements such as the Cantor alloy $(\mathrm{CoCrFeMnNi})$ could lead to single phase HEAs [8]. The prevalent rational explaining the formation of these multi-component single phase alloys considers that the configurational entropy increases with the number of components and is maximized at equimolarity, promoting in this way the formation of the solid-solution phase [2, 8, 10, 11]. The factors promoting the formation of HEAs are still heavily discussed in the literature. From a more applied point of view, being able to understand the formation of disordered solid solutions would enable the prediction of specific combinations of elements that tend to form single-phase HEAs. This would help navigate more efficiently the gigantic space of potential HEAs. To investigate phase stability and phase formation in HEAs, atomic descriptors such as electronegativity or valence electron concentration have been used [12, 13, 14, 15, 16, 17] as well as thermodynamics modeling based on experimental data and the CALPHAD approach [11, 18, 19]. Approaches based on descriptors rely on the appropriateness of the chosen descriptors while CALPHAD-built phase diagrams rely on experimental data that can be sparse. On the other hand, ab initio methods have been extremely successful in predicting formation enthalpies of materials and alloys [20, 21, 22].

In this work, we use a large database of ab initio computations to assess the reasons for the stability of HEAs. A regular solution model based on binary interactions fitted on ab initio computed enthalpies is developed together with a database of formation enthalpies covering more than $100000 \mathrm{BCC}$ and FCC equimolar compositions (up to quinaries) resulting from the combinations of 27 common elements. This database is used to statistically assess how the HEAs thermodynamic stability (based on both contribution of enthalpy and entropy) changes as the number of elements increases, offering a rationalization of the stability of these unique alloys.

At a given temperature and pressure, the thermodynamic stability of an alloy results from the competition between the Gibbs free energies of all possible phases. The Gibbs free energy $(G)$ of a phase depends on its enthalpy $(H)$ and entropy $(S)$ following $G=H-T S$. Assuming that configurational effects dominate the entropy and can be approximated by an ideal mixing, the molar entropy of an $n$-component alloy is $S_{\text {ideal }}=$ $-R \sum_{i}^{n} x_{i} \ln x_{i}$, where $R$ is the gas constant and $x_{i}$ the molar fraction of the $i$ th element. In the case of an equimolar alloy, the ideal mixing entropy becomes $S_{\text {ideal }}=R \ln n$. Figure 1 presents the evolution of the ideal entropy $\left(-\mathrm{T} S_{\text {ideal }}\right)$ as a function of the number of elements $n$. As the number of species in the alloy increases, the entropy contribution grows.

This simple analysis of high configurational entropy overcoming the enthalpy and stabilizing single phase multi-component solid solutions is still present in the literature even though it shows some major limitations. Indeed, since the configurational entropy monotonically increases with the number of elements, this rationale predicts that there will always be a number of species for which entropy overcomes enthalpy. It is contradicted by the experimental results [23, 24] that show a decrease of the number of single phase HEAs with the number of 


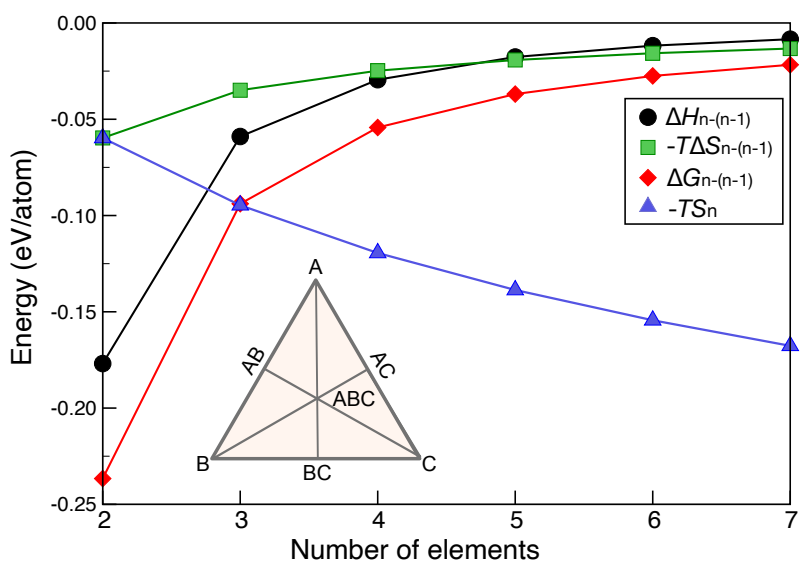

Figure 1: Dependence of thermodynamic quantities with the number of species in the solid solution. A temperature of $1000 \mathrm{~K}$ was assumed for all quantities. In addition to the entropy with respect to elements $-T S_{n}$ (blue triangles), we show the energy difference between the solid solution with $n$ elements and the competing solid solutions with $n-1$ elements for the entropy $-T \Delta S_{n-(n-1)}$ (green squares), the (average) enthalpy $\Delta H_{n-(n-1)}$ (black circles), and the (average) free energy $\Delta G_{n-(n-1)}$ (red diamonds). The energy difference of enthalpy of mixing is determined using Eq. 4, whereas the binary interaction $\bar{\Omega}$ is calculated using Eq. 3] and averaged over all binary systems. The phase diagram in the inset demonstrates the competition between phases containing different number of elements.

elements [11]. As previously mentioned in the literature [8, 11, 25], it can be argued that this simple analysis is in fact misleading. Indeed, the equilibrium state of an alloy results from the comparison of the Gibbs free energies of all the potential phases. It is thus of primary importance to compare the different possibilities. In that sense, when the ideal configurational entropy of an $n$ elements alloy increases, the entropy of the other competing alloys (with the number of elements lower than $n$ ) increases as well. Therefore, in the case of equimolar alloys, the true estimation of the entropy contribution to stability of an $n$ components solid solution is its difference with the entropy of the $n-1$ solid solutions. For instance, a ternary solid solution ABC competes directly with the formation of three binary solid solutions AB, AC, BC (see the inset of Fig. 1). The difference in entropy between the $n$ and $n-1$ components solid solutions can be shown to be (see Supplementary Materials 1)

$$
\Delta S_{n-(n-1)}=R \ln \left(\frac{n}{n-1}\right) .
$$

The quantity $\Delta S_{n-(n-1)}$ can be referred to as the ideal entropy of competition. Figure 1 shows how $-T \Delta S_{n-(n-1)}$ evolves with the number of elements in the alloy. Its evolution is drastically different from the ideal configurational entropy $\left(-T S_{\text {ideal }}\right)$. Even though both $S_{\text {ideal }}$ and $\Delta S_{n-(n-1)}$ favor mixing, the driving force for mixing in the case of $\Delta S_{n-(n-1)}$ decreases with an increasing number of elements (tending to zero for an infinite number of elements). This evolution is more physically intuitive as it is unlikely that the stabilization due to the entropy increases indefinitely with the number of elements.

While the ideal configurational entropy is the same for any equimolar alloy with the same number of elements, the enthalpy of mixing depends on the nature of the constitutive elements. The enthalpies of random solid solutions can be calculated by density functional theory (DFT) using the special quasi random structures (SQS) approach [26] which provides a series of representative structures on a given lattice that are the closest to a random solid solution. This SQS approach thus offers the possibility to build a database of enthalpies of equimolar random solid solutions using DFT. However, this task remains formidable. Indeed, the 27 common elements (Al, Co, Cr, $\mathrm{Cu}, \mathrm{Fe}, \mathrm{Hf}, \mathrm{Mn}, \mathrm{Mo}, \mathrm{Nb}, \mathrm{Ni}$, Ta, Ti, W, Zr, V, Mg, Re, Os, Rh, Ir, Pd, Pt, Ag, Au, Zn, Cd, Hg), would give rise 
to 2925 ternary, 17550 quaternary, and 80730 quinary equimolar random solutions. For each of these alloys, the pertinent SQS needs to be computed. Alternatively, the regular solution method is widely used. The enthalpy of mixing of an equimolar $n$-component alloy system on a given lattice (e.g.m, FCC or BCC) can be described by the binary interactions $\left(\Omega_{i j}\right)$ as

$$
\Delta H_{n}^{\mathrm{mix}}=\sum_{i} \sum_{j>i} \Omega_{i j} x_{i} x_{j}=\sum_{i} \sum_{j>i} \Omega_{i j}\left(\frac{1}{n}\right)^{2},
$$

where the atomic fraction $x_{i}=x_{j}=\frac{1}{n}$, and the sum over $i$ and $j$ runs through all combinations of two elements and $\Omega_{i j}$ are interaction terms related physically to the bonding of those two elements. For instance, the enthalpy of a quaternary alloy $\mathrm{A}_{0.25} \mathrm{~B}_{0.25} \mathrm{C}_{0.25} \mathrm{D}_{0.25}$ would be the result of 6 binary interaction terms $\Omega_{A B}, \Omega_{B C}, \Omega_{C D}$, $\cdots$, weighted equally. The binary interaction parameters were obtained from the enthalpy of mixing of the binary systems $\Delta H_{i j}^{\operatorname{mix}}$ as

$$
\Omega_{i j}=\left(\frac{1}{x_{i} x_{j}}\right) \Delta H_{i j}^{\mathrm{mix}}=4\left[E_{i j}^{\mathrm{SQS}}-\frac{1}{2}\left(E_{i}+E_{j}\right)\right],
$$

where $x_{i}=x_{j}=\frac{1}{2}$ for an equimolar binary. $E_{i j}^{\mathrm{SQS}}$ is the DFT total energy of the binary SQS, and $E_{i}$ is the total energy of the constituent unary system $i$ with the same parent lattice of the SQS. For each binary, we consider the permutation in the atomic positions of the two elements and take the average total energies of the two SQS structures for $E_{i j}^{\mathrm{SQS}}$. The SQS were obtained using the Alloy Theoretic Automated Toolkit (ATAT) software [27]. The pair interactions for the SQS structures have been set up to the 5th nearest neighbor while the triplet interactions have been chosen to be up to the 3rd nearest neighbor. DFT calculations were performed with the generalized-gradient approximation within the Perdew-Burke-Ernzerhof (GGA-PBE) implementation using the Vienna ab initio simulation package (VASP) [28, 29]. Enthalpies are considered equal to the DFT total energies as the pressure times volume component is small in solids and can be neglected. The magnetic moments were initialized in a ferromagnetic state for magnetic elements. A grid density of $100 \mathrm{k}$-points $/ \AA^{-3}$ was considered. The plane-wave energy cutoff was set to $400 \mathrm{eV}$. The atomic positions were considered relaxed when the forces on each atom were less than $0.02 \mathrm{eV} / \AA \AA$. All input generation and analysis including the convex hull constructions were performed using the pymatgen software [30, 31].

We found that a simple regular solution with only binary interactions reproduces very well the enthalpies obtained from the full SQS of a series of quinary and quaternary systems. Figure 2(a) compares the enthalpy of some quaternary and quinary FCC and BCC systems computed with our regular solution model to the enthalpy of the same alloy computed by the full SQS. The agreement is reasonably good despite the simplicity of the model. The adequacy of the proposed approach can also be seen in Fig. 2(b) which compares the binary regular solution model enthalpy with the full SQS enthalpy for the FCC and BCC alloys ( $R^{2}$ value of 0.977). The root mean square error from the regular solution model compared to the full SQS is around $16 \mathrm{meV} /$ atoms. This value is in the order of magnitude of the typical DFT errors as well as other contributions neglected in this work such as the vibrational entropy [21, 32, 33, 34]. We also tested a ternary regular solution model and no significant improvement with respect to the full SQS model was observed as the root mean square error was not further improved (see Supplementary Materials 2).

From this analysis, it can be concluded that for the enthalpy of multi-component random solid solutions it is sufficient to use the enthalpy of binary SQS with the regular solution model. In total, only 1404 computations of 

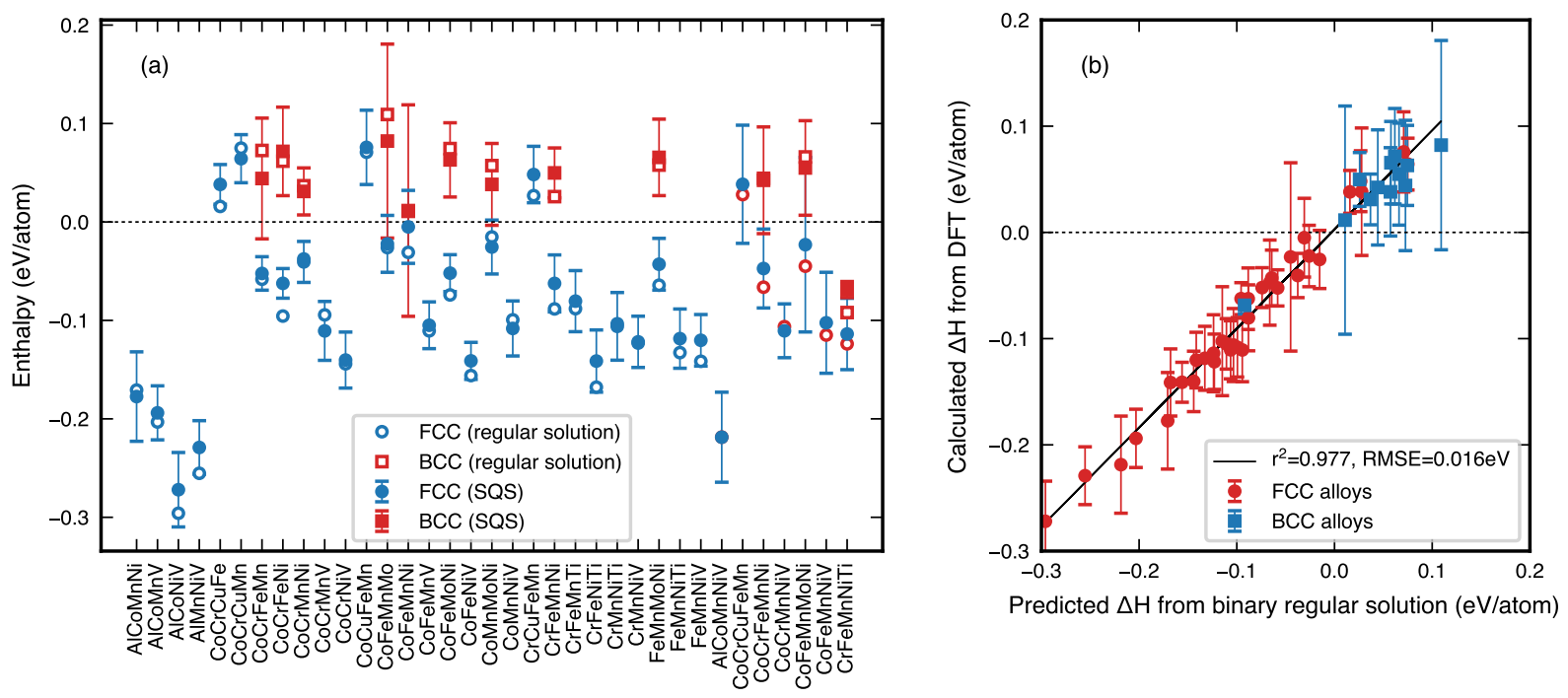

Figure 2: (a) Comparison between the enthalpy value obtained by the binary regular solution model and calculated with full SQS for quaternary and quinary alloys. (b) Correlation between the enthalpy values obtained by the binary regular solution model [cf. Eqs. 22] and (3)] and calculated through SQS for the FCC (in red) and BCC (in blue) alloys. For both plots the bar indicates the spread in energy between all the different SQS.

SQS of 16 atoms had to be performed to generate a database covering all 101205 ternary, quaternary, and quinary combinations of the 27 elements for both BCC and FCC structures. Owing to the regular solution model, it is now possible to investigate how the enthalpy competition between $n$ components equimolar alloys and their lower equimolar $n-1$ components alloys evolves as the number of components increases. As the enthalpy depends on the nature of the elements, the proposed analysis is statistical and is based on the database of ab initio estimated binary interactions. It is found that the competition between the enthalpy of mixing of the $n$ element equimolar alloy and that of its lower $n-1$ element equimolar alloys is (see Supplementary Materials 3):

$$
\Delta H_{n-(n-1)}=\frac{\overline{\boldsymbol{\Omega}}}{2 n(n-1)},
$$

where $\bar{\Omega}$ is the average of the different binary interaction terms in the database of the FCC or BCC alloys. The enthalpic competition is plotted in Fig. 1. This term is always negative in our dataset, indicating a general tendency for mixing of any number of components. In other words, mixing in random solid solutions is overall favored for FCC or BCC lattices. However, the magnitude of the enthalpic driving force varies strongly with the number of elements. It decreases as the number of species in the alloy increases, tending towards zero for increasing number of elements. Finally, the evolution of the Gibbs free energy with the number of species at a representative synthesis temperature of $1000 \mathrm{~K}$ is plotted in Fig. 1 by adding the enthalpic and entropic contributions. The tendency present both in the entropy and enthalpy contributions of a favored mixing which becomes weaker with the number of elements is of course also present in the resulting Gibbs free energy evolution.

So far, we have analyzed trends in stability assuming that an $n$-component alloys only competes with the $n-1$ component alloys. However, the enthalpy of an $n$-component alloys is not only in competition with the $n-1$ alloys but also with the $n-2$ or lower orders alloys. Fortunately, a full stability analysis taking into account all competing equimolar solid solutions can be performed using the convex hull construction. The convex hull construction effectively compares all the possible decomposition for a series of phases and indicates the stable 


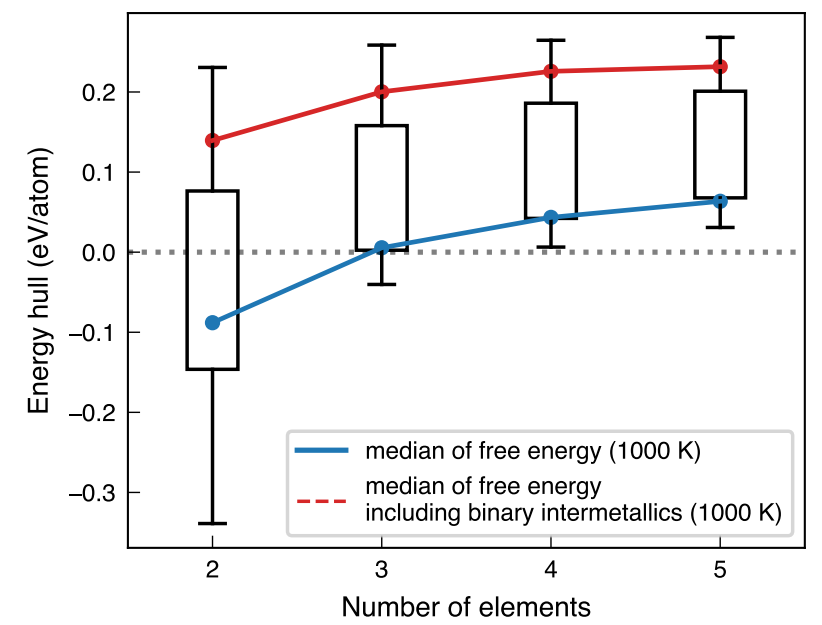

Figure 3: Free energy and enthalpy relative to the energy hull for all equimolar FCC solid solutions. A negative value indicates stability (energy below hull) and a positive value indicates instability (energy above hull). The black boxes represent the distribution of the enthalpy relative to the hull. These boxes correspond to the first and third quartile while the bars correspond the 90th and 10th percentiles. The blue and red lines plot the median of the free energy relative to the hull at $1000 \mathrm{~K}$ considering only solid solutions and including intermetallics, respectively.

phase or the combinations of phases (see e.g., Ref. [21]). This full stability analysis for all possible alloys up to 5 elements based on 27 elements (which represents a total 101205 equimolar compositions) was performed based on the regular solution enthalpy and the ideal configurational entropy. For each alloy, a convex hull analysis was performed to show how stable or unstable the equimolar alloy is with respect to all competing lower orders equimolar solid solutions. For example, for the quinary alloy $A_{0.2} B_{0.2} C_{0.2} D_{0.2} E_{0.2}$, the competition with respect to 10 binaries $\left(A_{0.5} B_{0.5}, A_{0.5} C_{0.5}, \cdots\right), 10$ ternaries $\left(A_{1 / 3} B_{1 / 3} C_{1 / 3}, A_{1 / 3} B_{1 / 3} D_{1 / 3}, \cdots\right)$, and 5 quaternaries $\left(A_{0.25} B_{0.25} C_{0.25} D_{0.25}, A_{0.25} B_{0.25} C_{0.25} E_{0.25}, \cdots\right)$ was considered. The results of this convex hull analysis are presented in Fig. 3 The enthalpy above hull (positive) and the inverse enthalpy above hull (negative) are reported. Note that a positive value indicates an instability with respect to competing phases and a negative value indicates a stable phase. The sizable spread of the enthalpy is expected from a strong dependence of the enthalpy of mixing on the nature of the elements. The enthalpy above/below hull increases as the number of elements increases. The strongest formers are binary alloys with the higher orders alloys for which the enthalpic driving force for mixing become increasingly unfavorable. By adding an ideal configurational entropy component at a temperature representative of alloy processing $(1000 \mathrm{~K})$, we can compute a similar convex hull using the Gibbs free energy. The median values of the free energy above/below hull is plotted in Fig. 3 The Gibbs free energy is shifted towards higher stability (more negative values) compared to the enthalpy as expected from the entropic stabilization. We note that the trends are very similar for both the FCC and BCC lattices (see Supplementary Materials 4 and 5). With this stability analysis, the proportion of quinary systems that would be thermodynamically stable at $1000 \mathrm{~K}$ can be estimated. Figure 4 plots the percentage of equimolar compositions that would thermodynamically form a single-phase HEA in our model at $1000 \mathrm{~K}$ as a function of the number of elements. About $20 \%$ (16469 alloys) and $14.5 \%$ (11 729 alloys) of the quinary alloys are found to be thermodynamically stable at $1000 \mathrm{~K}$ for the FCC and the BCC lattices, respectively. This indicates that randomly mixing elements up to quinary alloys on simple FCC 


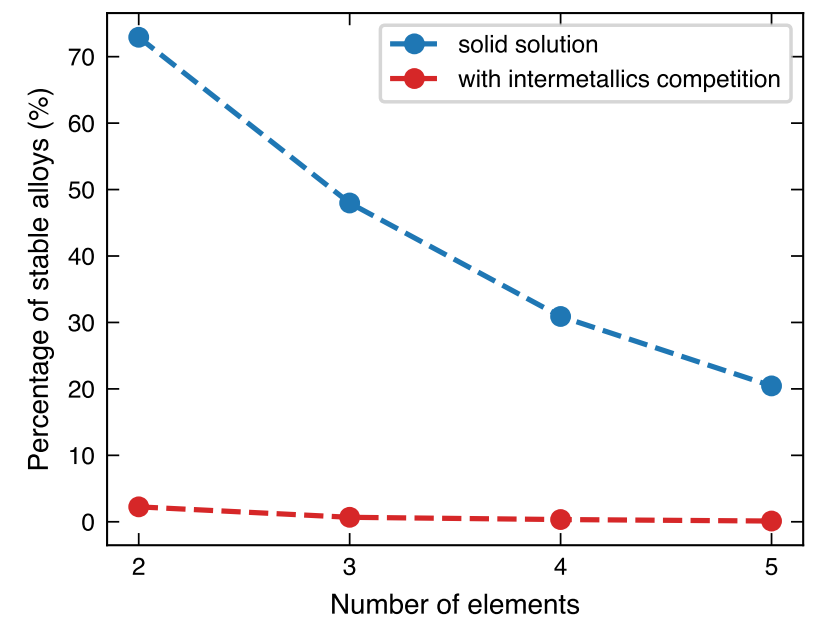

Figure 4: Percentage of stable FCC (blue points) without and with the intermetallic alloys (red points) versus the number of elements at $1000 \mathrm{~K}$.

and BCC lattices appears to be not strongly prevented by energetic factors, i.e., the penalty from the enthalpy term is not very large. It is worth noting that this easy mixing is likely to be strongly related to the nature of the metallic bond. Compared to the experimental literature, the number of potential multi-component stable alloys appears to be relatively high in view of the limited number of reported HEAs (less than 300 quaternary and quinary equimolar alloys) [35, 36]. This suggests that other factors beyond competitions between random solid solutions also prevent the formation of HEAs. Indeed, intermetallics have hitherto not been included in our analysis. In order to assess the importance of intermetallics, the enthalpy of the stable binary intermetallics were calculated by DFT and a new convex hull analysis was performed including the competition from intermetallics. The intermetallic structures were obtained from the AFLOW database [37]. Since the parameters of present DFT calculations differ from what is used in the AFLOW database, we selected intermetallic structures with an energy-above-hull value smaller than $10 \mathrm{meV} /$ atom from the database and performed a full relaxation for these structures. Figure 3 shows the free energy below/above hull including binary intermetallics. The free energy is very significantly shifted towards higher values and thus stronger instability. This shift in free energy strongly impacts the percentage of stable HEAs as shown in Fig. 4. This indicates that the rare combination of elements leading to HEA formation requires both a favorable enthalpic mixing of the elements on a given FCC or BCC lattice (i.e., a tendency to easily form solid solutions) and the absence of too strongly forming intermetallics.

We note that our present analysis does not consider the temperature dependence of the enthalpy and the entropy. In addition, we consider only the ideal configurational component of the entropy as it is at the root of the regular solution model. Taking into account the temperature effect [38, 39, 40, 41, 42, 43, 44, 45, 46, 47, 48] and, in particular, the vibrational entropy [49, 50, 51] would be computationally too expensive for the present large-scale study. Nevertheless, we show that the analysis based on the simple regular solution model and ideal configurational entropy captures the statistical trend on how the increasing number of elements stabilizes the formation of single-phase HEAs.

In conclusion, motivated by the need to understand the fundamental thermodynamic reasons behind HEAs formation, we studied how the enthalpy and entropy of multi-component equimolar random solid solutions change 
with an increasing number of elements. We demonstrated that the original rational explaining the existence of HEAs through an increase of ideal configurational entropy with the number of elements is misleading. A more relevant analysis based on entropy differences between competing solid solutions (e.g., quinary versus quaternary) indicates a lower entropic driving force when the number of elements increases. The second part of our analysis considered the enthalpic contribution using ab initio computations. We showed that a simple regular binary solution model using ab initio computed binary SQS leads to an adequate accuracy in computing the enthalpy of multi-component alloys. Using this approach, a database of more than 100000 alloys based on FCC and BCC random solid solutions was constructed. The enthalpic contribution is overall favorable to solid solution formation, but with a large spread due to chemistry. As the number of elements increases, the enthalpic driving force for mixing becomes weaker. When combining enthalpy and entropy at a relevant processing temperature, we highlight that adding more elements makes it statistically more difficult to form random solid solutions. Nevertheless, our analysis shows that the mixing of elements in equimolar random solution is still favorable enough to lead to tens of thousands of potential HEAs stable at a reasonable processing temperature of $1000 \mathrm{~K}$. However, when competing intermetallics are taken into account, the number of potential stable HEAs is significantly limited. Therefore, in addition to maximizing the configurational entropy, the formation of a single-phase HEA further requires a chemistry that do not present strongly competitive intermetallics.

\section{Acknowledgments}

The research was funded by the Walloon Region under the agreement No. 1610154-EntroTough in the context of the 2016 Wallinnov call. Computational resources were provided by the supercomputing facilities of UCLouvain and the Consortium des Equipements de Calcul Intensif en Fédération Wallonie-Bruxelles (CECI) funded by the Fonds de la Recherche Scientifique de Belgique (FNRS).

\section{References}

[1] B. Cantor, I. T. Chang, P. Knight, A. J. Vincent, Mater. Sci. Eng., A 375-377 (2004) 213-218.

[2] J. W. Yeh, S. K. Chen, S. J. Lin, J. Y. Gan, T. S. Chin, T. T. Shun, C. H. Tsau, S. Y. Chang, Adv. Eng. Mater. 6 (5) (2004) 299-303.

[3] D. B. Miracle, Mater. Sci. Technol. 31 (10) (2015) 1142-1147.

[4] B. Gludovatz, A. Hohenwarter, D. Catoor, E. H. Chang, E. P. George, R. O. Ritchie, Science 345 (6201) (2014) 1153-1158.

[5] S. Gorsse, D. B. Miracle, O. N. Senkov, Acta Mater. 135 (2017) 177-187.

[6] O. N. Senkov, S. Gorsse, D. B. Miracle, Acta Mater. 175 (2019) 394-405.

[7] B. Gwalani, S. Gorsse, D. Choudhuri, Y. Zheng, R. S. Mishra, R. Banerjee, Scr. Mater. 162 (2019) 18-23.

[8] D. B. Miracle, O. N. Senkov, Acta Mater. 122 (2017) 448-511.

[9] S. Gorsse, J. P. Couzinié, D. B. Miracle, C. R. Phys. 19 (8) (2018) 721-736. 
[10] F. Otto, Y. Yang, H. Bei, E. P. George, Acta Mater. 61 (7) (2013) 2628-2638.

[11] S. Gorsse, F. Tancret, J. Mater. Res. (2018) 1-25.

[12] Y. Zhang, Y. J. Zhou, J. P. Lin, G. L. Chen, P. K. Liaw, Adv. Eng. Mater. 10 (6) (2008) 534-538.

[13] M. G. Poletti, L. Battezzati, Acta Mater. 75 (2014) 297-306.

[14] Y. F. Ye, C. T. Liu, Y. Yang, Acta Mater. 94 (2015) 152-161.

[15] Y. F. Ye, Q. Wang, J. Lu, C. T. Liu, Y. Yang, Scr. Mater. 104 (2015) 53-55.

[16] M. C. Troparevsky, J. R. Morris, P. R. Kent, A. R. Lupini, G. M. Stocks, Phys. Rev. X 5 (1) (2015) 1-6.

[17] S. Guo, C. Ng, J. Lu, C. T. Liu, J. Appl. Phys. 109 (10) (2011).

[18] O. N. Senkov, J. D. Miller, D. B. Miracle, C. Woodward, Nat. Commun. 6 (2015) 1-10.

[19] S. Gorsse, O. N. Senkov, Entropy 20 (12) (2018) 1-9.

[20] S. Curtarolo, D. Morgan, G. Ceder, CALPHAD 29 (3) (2005) 163-211.

[21] G. Hautier, S. P. Ong, A. Jain, C. J. Moore, G. Ceder, Phys. Rev. B 85 (15) (2012).

[22] Y. Sun, Y. Zhang, F. Zhang, Z. Ye, Z. Ding, C.-Z. Wang, K.-M. Ho, J. Appl. Phys. 120 (1) (2016) 015901.

[23] S. Gorsse, M. Nguyen, O. Senkov, D. Miracle, Data in Brief 3 (2018) 1-15.

[24] P. Zhang, J. J. Maldonis, M. Besser, M. Kramer, P. M. Voyles, Acta Mater. 109 (2016) 103-114.

[25] F. Zhang, C. Zhang, S. L. Chen, J. Zhu, W. S. Cao, U. R. Kattner, CALPHAD 45 (2014) 1-10.

[26] A. Zunger, S. H. Wei, L. G. Ferreira, J. E. Bernard, Phys. Rev. Lett. 65 (3) (1990) 353-356.

[27] A. Van de Walle, M. Asta, G. Ceder, CALPHAD 26 (4) (2002) 539-553.

[28] G. Kresse, J. Furthmüller, Phys. Rev. B 54 (16) (1996) 11169-11186.

[29] G. Kresse, Phys. Rev. B 59 (3) (1999) 1758-1775.

[30] S. P. Ong, W. D. Richards, A. Jain, G. Hautier, M. Kocher, S. Cholia, D. Gunter, V. L. Chevrier, K. A. Persson, G. Ceder, Comput. Mater. Sci 68 (2013) 314-319.

[31] A. Jain, G. Hautier, C. J. Moore, S. Ping Ong, C. C. Fischer, T. Mueller, K. A. Persson, G. Ceder, Comput. Mater. Sci 50 (8) (2011) 2295-2310.

[32] J. Neuhaus, M. Leitner, K. Nicolaus, W. Petry, B. Hennion, A. Hiess, Phys. Rev. B 89 (18) (2014) 1-6.

[33] A. Manzoor, S. Pandey, D. Chakraborty, S. R. Phillpot, D. S. Aidhy, npj Comput. Mater. 4 (1) (2018) 1-10.

[34] G. Petretto, X. Gonze, G. Hautier, G. M. Rignanese, Comput. Mater. Sci 144 (2018) 331-337.

[35] O. N. Senkov, D. B. Miracle, K. J. Chaput, J. P. Couzinie, J. Mater. Res. 33 (19) (2018) 3092-3128. 
[36] M. Vaidya, G. M. Muralikrishna, B. S. Murty, J. Mater. Res. 34 (5) (2019) 664-686.

[37] R. H. Taylor, F. Rose, C. Toher, O. Levy, K. Yang, M. Buongiorno Nardelli, S. Curtarolo, Comput. Mater. Sci 93 (2014) 178-192.

[38] T.-H. Gao, X. Hu, Q. Xie, Y. L. Lei Ren, Mater. Res. Express (2017) 0-16.

[39] Y. Ikeda, B. Grabowski, F. Körmann, Mater Charact 147 (2019) 464-511.

[40] F. Tian, L. K. Varga, N. Chen, L. Delczeg, L. Vitos, Phys. Rev. B 87 (7) (2013).

[41] P. Zhao, J. Li, J. Hwang, Y. Wang, Acta Mater. 134 (2017) 104-115.

[42] M. Fedorov, J. S. Wróbel, A. Fernández-Caballero, K. J. Kurzydłowski, D. Nguyen-Manh, Phys. Rev. B 101 (2020) 174416.

[43] X. Sun, H. Zhang, S. Lu, X. Ding, Y. Wang, L. Vitos, Acta Mater. 140 (2017) 366-374.

[44] L. Li, Q. Luo, R. Li, H. Zhao, K. W. Chapman, P. J. Chupas, L. Wang, H. Liu, Sci. Rep. 7 (2017) 46762.

[45] M. Widom, J Mater Res 33 (19) (2018) 2881-2898.

[46] H. Ge, H. Song, J. Shen, F. Tian, Mater Chem Phys 210 (2018) 320-326.

[47] B. Wang, X. Huang, A. Fu, Y. Liu, B. Liu, Mater. Sci. Eng., A (2018).

[48] S. Luo, L. Vitos, C. Zhao, Y. Su, Z. Wang, Comput. Mater. Sci. 186 (2021) 110021.

[49] Z. Ma, Y. Guo, L. T. Li Yeung, P. Gao, Z. Yang, X. Zeng, J. Alloys Compd. 648 (2015) 18-21.

[50] F. Körmann, Y. Ikeda, B. Grabowski, M. H. F. Sluiter, npj Comput. Mater. 3 (1) (2017) 36.

[51] B. Grabowski, Y. Ikeda, P. Srinivasan, F. Körmann, C. Freysoldt, A. I. Duff, A. Shapeev, J. Neugebauer, npj Comput. Mater. 5 (1) (2019) 80. 